\title{
Vacuum Stress-Energy Density and Its Gravitational Implications
}

\author{
Ricardo Estrada ${ }^{1}$, Stephen A Fulling ${ }^{2}$, Lev Kaplan ${ }^{3}$, Klaus \\ Kirsten $^{4}$, Zhonghai Liu ${ }^{5}$ and Kimball A Milton ${ }^{6}$ \\ ${ }^{1}$ Department of Mathematics, Louisiana State University, Baton Rouge, LA, \\ 70803-4918 USA \\ 2 Department of Mathematics, Texas A\&M University, College Station, TX, \\ 77843-3368 USA \\ ${ }^{3}$ Department of Physics, Tulane University, New Orleans, LA, 70118 USA \\ ${ }^{4}$ Department of Mathematics, Baylor University, Waco, TX, 76798-7328 USA \\ ${ }^{5}$ Department of Physics, Texas A\&M University, College Station, TX, 77843-4242 \\ USA \\ ${ }^{6}$ Department of Physics and Astronomy, University of Oklahoma, Norman, OK, \\ 73019-2061 USA
}

E-mail: fulling@math.tamu.edu

\begin{abstract}
In nongravitational physics the local density of energy is often regarded as merely a bookkeeping device; only total energy has an experimental meaning - and it only modulo a constant term. But in general relativity the local stressenergy tensor is the source term in Einstein's equation. In closed universes, and those with Kaluza-Klein dimensions, theoretical consistency demands that quantum vacuum energy should exist and have gravitational effects, although there are no boundary materials giving rise to that energy by van der Waals interactions. In the lab there are boundaries, and in general the energy density has a nonintegrable singularity as a boundary is approached (for idealized boundary conditions). As pointed out long ago by Candelas and Deutsch, in this situation there is doubt about the viability of the semiclassical Einstein equation. Our goal is to show that the divergences in the linearized Einstein equation can be renormalized to yield a plausible approximation to the finite theory that presumably exists for realistic boundary conditions. For a scalar field with Dirichlet or Neumann boundary conditions inside a rectangular parallelepiped, we have calculated by the method of images all components of the stress tensor, for all values of the conformal coupling parameter and an exponential ultraviolet cutoff parameter. The qualitative features of contributions from various classes of closed classical paths are noted. Then the Estrada-Kanwal distributional theory of asymptotics, particularly the moment expansion, is used to show that the linearized Einstein equation with the stress-energy near a plane boundary as source converges to a consistent theory when the cutoff is removed.
\end{abstract}

PACS numbers: 03.70.+k, 04.20.Cv, 11.10.Gh, 45.20.Lc 
This paper reports work in progress on a project combining researchers in Texas, Louisiana, and Oklahoma. It is supported by NSF Grants PHY-0554849 and PHY0554926 .

\section{General remarks}

\subsection{Localization of energy}

In electrostatics there are two well known integral representations of the total energy of a system:

$$
E=\frac{1}{2} \int \rho V d^{3} x=\frac{1}{2} \int \mathbf{E}^{2} d^{3} x
$$

The integrands are different: $\rho(\mathbf{x}) V(\mathbf{x}) \neq \mathbf{E}(\mathbf{x})^{2}$. Thus (1) does not commit us to a physical concept of energy density. It is common to regard the integrand as merely a matter of bookkeeping, or even to regard the $\rho V$ form as more fundamental. General relativity, however, requires us to take the concept of a local energy density seriously. The central dynamical equation of Einstein is

$$
R_{\mu \nu}-\frac{1}{2} R g_{\mu \nu}=T_{\mu \nu}
$$

and for electromagnetism the 00 component of the stress tensor $T_{\mu \nu}$ is, in the flat-space limit,

$$
T_{00}(\mathbf{x})=\frac{1}{2}\left(\mathbf{E}^{2}+\mathbf{B}^{2}\right)
$$

If the source is a point particle, some renormalization is already necessary at this classical level.

When the matter in the model includes quantum fields, in principle their quantum vacuum energy must be included in $T_{00}$.

\subsection{Reality of vacuum energy}

Vacuum energy as a scientific subject began with the study of the van der Waals attraction between polarizable atoms.

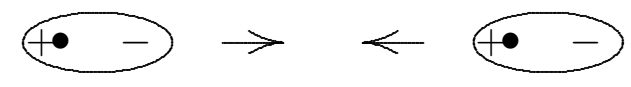

Casimir and Polder turned attention to the interaction between such an atom and a conducting plate.

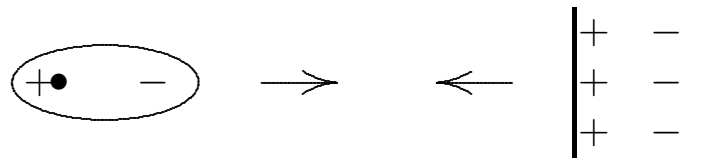

Then Casimir simplified the situation further to two parallel conducting plates

$$
\begin{array}{ll}
+ & - \\
+ & - \\
+ & -
\end{array}|<\quad| \begin{array}{ll}
+ & - \\
+ & - \\
+ & -
\end{array}
$$


and found that the calculation was most easily done by ignoring the charges in the plates in favor of the energy of the electromagnetic field in the gap. At that point it was still possible to assert that the vacuum energy was a mere bookkeeping device, the real physics being the van der Waals interaction between the electrons in the plates. However, in the 1970s came the first renaissance of Casimir energy in cosmology. The reasoning that applied to a field with perfect-conductor (or Dirichlet) boundary conditions surely applied equally to a field with periodic boundary conditions: in a closed universe the negative vacuum energy of a quantum field would give rise to a force tending to make the universe contract.

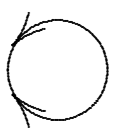

The polarizable atoms and electrons have now disappeared! The cosmological Casimir force, if it indeed exists, is something intrinsic to the field, not an indirect representation of the interaction of (now nonexistent) boundary bodies. Yet to doubt the existence of this force is to change one's physical interpretation of the quantum field theory in an ad hoc way when moving from one scenario to another.

In the decade of the 2000s we are seeing the second cosmological renaissance of vacuum energy. The notorious "dark energy" calls out for explanation, and the Casimir energy associated with Kaluza-Klein dimensions may be relevant [1,2]. Vacuum energy should also play a role in scenarios with parallel branes (e.g., [3]).

\subsection{Boundaries}

We return now to the mundane situation of idealized boundaries (conductors, or Dirichlet boundaries for a scalar field) in flat space. The energy density then has nonintegrable singularities near the boundaries:

$$
T_{00} \sim \frac{c_{1}}{s^{4}}+\frac{c_{2}}{s^{3}}+\cdots,
$$

where $s$ is the distance from the boundary [4]. (There are finite results for the electromagnetic field in certain special geometries, but these are apparently fortuitous and without fundamental significance.) Zeta-function regularization magically removes these infinities from the total energy, except in some cases where the zeta function has a pole. Ultraviolet-cutoff regularization requires them to be discarded ad hoc (with a logarithmic ambiguity — discussed further below - in those cases where the zeta function has a pole).

The standard response to this situation is to note that for more realistic boundary conditions these boundary effects would be (possibly large but) finite. They are part of the energy of the boundary material. It seems now to be agreed that the naively renormalized theory is reasonable for calculating forces between rigid bodies, where the cutoff-dependent terms cancel between the configurations being compared in an infinitesimal displacement (e.g., [5]). For deformable bodies, where such cancellation 
generally does not take place, the physics of the material itself must be included anyway and may be much more significant than the vacuum effect [6].

But what about gravity? As Deutsch and Candelas [4] pointed out, even when the total energy is finite (say, because of cancellations between the exterior and interior of a thin shell), the vacuum energy (3) cannot be ignored in the Einstein equation (2). It is this puzzle that we are trying to address.

Gravitational effects in the lab are formally infinite but presumably actually tiny. Therefore, it should be sufficient to treat the gravitational field through the linearized Einstein equation. We take a flat background, although at a more sophisticated stage one might need to add a small curvature caused by the mass of the boundary material. Our working hypothesis is that the stress tensor calculated for idealized boundary conditions with the ultraviolet cutoff parameter kept finite is a reasonable ad hoc model for the true situation. The theory will have a sensible renormalized limit when the cutoff is taken away. This requires making sense of the Einstein equation with a distributional source. To define the distributions involved, the functions that arise in the source and in the solutions of the equation must be "regularized" in the mathematicians' sense (which has more to do with renormalization than with regularization as physicists use those terms).

\section{Formalism}

\subsection{Scalar field and stress tensor}

We follow the sign conventions of [7] (in which $g_{00}<0$ but $T_{00}>0$ for normal matter). We consider the standard scalar field with action

$$
S=\int_{\Omega} L \sqrt{g} d^{d+1} x+\text { boundary term, } \quad L=\frac{1}{2}\left[g^{\mu \nu} \partial_{\mu} \phi \partial_{\nu} \phi+\xi R \phi^{2}\right] .
$$

The stress tensor then is

$$
T^{\mu \nu}=\frac{2}{\sqrt{g}} \frac{\delta S}{\delta g_{\mu \nu}},
$$

and in the flat-space limit the field equation is

$$
\frac{\partial^{2} \phi}{\partial t^{2}}=\nabla^{2} \phi \quad \text { with boundary conditions } \equiv-H \phi
$$

In (4) $R$ is the curvature scalar and $\xi$ labels different possible gravitational couplings. In curved space different values of $\xi$ are different theories; after the reduction to flat space the field equation and (classical) total energy are independent of $\xi$, but the stress tensors are different.

The most important component of the stress tensor is the energy density. It is convenient to express the quantities for general $\xi$ in terms of those for $\xi=\frac{1}{4}$. One has

$$
T_{00}\left(\xi=\frac{1}{4}\right)=\frac{1}{2}\left[\left(\frac{\partial \phi}{\partial t}\right)^{2}-\phi \nabla^{2} \phi\right],
$$




$$
T_{\mu \nu}(\xi)=T_{\mu \nu}\left(\frac{1}{4}\right)+\Delta T_{\mu \nu}, \quad \Delta T_{00}=-2\left(\xi-\frac{1}{4}\right) \nabla \cdot(\phi \nabla \phi) .
$$

The fact that $\Delta T_{00}$ is a divergence accounts for the fact that $E=\int_{\Omega} T_{00} d^{d} x$ is independent of $\xi$. (When Robin boundaries [8, 9, 10] or delta potentials [11, 12] are present, nontrivial boundary terms nevertheless must be taken into account when $\xi \neq \frac{1}{4}$.)

\subsection{Ultraviolet cutoff and cylinder kernel}

In terms of the eigenvalues $\omega_{n}^{2}$ of $H$ the cut-off energy is

$$
\langle E\rangle_{t} \equiv \frac{1}{2} \sum_{n=1}^{\infty} \omega_{n} e^{-\omega_{n} t}=-\frac{1}{2} \frac{\partial}{\partial t} \sum_{n=1}^{\infty} e^{-\omega_{n} t} .
$$

Here $t$ is not time, but its mathematical role is similar to that of the time in the heat, wave, or Schrödinger kernel. There are similar formulas for all the components of $T_{\mu \nu}$. It is convenient to introduce the cylinder kernels defined by

$$
T(t, x, y) \equiv \sum_{n=1}^{\infty} \varphi_{n}(x) \varphi_{n}(y)^{*} e^{-t \omega_{n}}=\left\langle x\left|e^{-t \sqrt{H}}\right| y\right\rangle \equiv \frac{\partial \bar{T}}{\partial t} .
$$

Then

$$
\left\langle T_{00}\left(\xi=\frac{1}{4}\right)\right\rangle_{t}=-\frac{1}{2} \frac{\partial T}{\partial t}(t, x, x), \quad\langle E\rangle_{t}=-\frac{1}{2} \frac{\partial}{\partial t} \operatorname{Tr} T .
$$

To get the energy density for $\xi \neq \frac{1}{4}$ one needs $\bar{T}$, an indefinite integral of $T$ :

$$
\left\langle\Delta T_{00}\right\rangle_{t}=\left(\xi-\frac{1}{4}\right) \nabla_{x} \cdot\left[\nabla_{y} \bar{T}(t, x, y)\right]_{y=x} .
$$

(The Green function $\bar{T}$ is often introduced differently, either as the resolvent kernel in $\mathbf{R} \times \Omega$ with its source on $t=0$, or as an analytic continuation to imaginary time of the Wightman or Feynman 2-point function.)

\section{Rectangular parallelepipeds}

Rectangles and rectangular boxes are perhaps the most often studied configurations for vacuum energy; the references are too numerous to list. We believe, however, that there are still useful things to say about them. We are calculating local quantities (not just the total energy and the global density of states). Specifically, we find all components of $T_{\mu \nu}$, for all values of $\xi$, displaying the full dependence on the cutoff parameter $t$. Many of the results are complete for arbitrary dimension and either Dirichet or Neumann boundary conditions on each side of the box. Here we can provide only a brief survey.

The great attraction of rectangular geometries is that the multiple-reflection (image) method is exact in these cases. (In the long run, of course, this is also their great limitation; the problem of a general geometry is significantly different. Nevertheless, these models provide insight into what is happening at boundaries.) We construct the

energy density (and other components of the stress tensor) as a sum of contributions from closed and periodic paths. Consequently, there is some overlap with the work of 
Hertzberg et al. [13]. One difference is that we construct the cylinder kernel directly, not via the density of states.

A sum over classical paths inside the $d$-dimensional rectangular box (with specular reflection at the sides) is equivalent to an image sum in the covering space, $\mathbf{R}^{d}[14]$ :

\begin{tabular}{c|c|c|c|cl} 
& $\bullet$ & $\circ$ & $\bullet$ & $\circ$ & $\times=$ point $x$ under study, \\
& $\circ$ & $*$ & $\circ$ & $*$ & \\
& & & & $=$ periodically displaced image, \\
& $\times$ & $\circ$ & $\bullet$ & $\circ$ & $*=$ reflection through a side \\
\hline & $\circ$ & $*$ & $\circ$ & $*$ &
\end{tabular}

\subsection{Periodic paths}

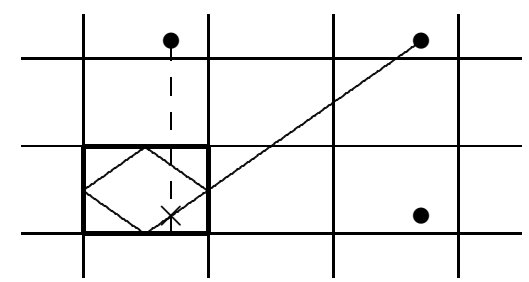

Periodic terms yield constant densities, independent of ( $\mathbf{x}$ and) $\xi$. Two typical periodic paths are shown here (dashed and undashed) and in each of the similar figures below.

\subsection{Corner paths}

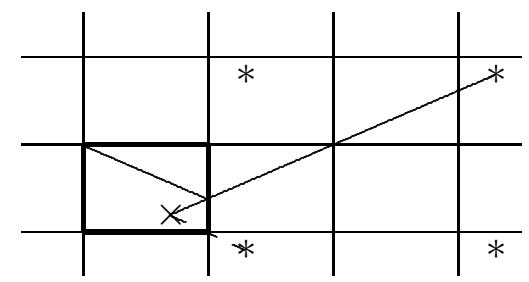

Regularized corner terms make no contribution to the total energy, although the energy density with the cutoff removed diverges as the corner is approached. That is, the limit $t \rightarrow 0$ is nonuniform, and it makes a huge difference whether it is taken before or after the spatial integration. This is a generalization of an observation of Ford and Svaiter [15]; we reported the one-dimensional case at the 2003 QFExt [16], and we refer to those proceedings for numerical plots that make this point clear.

Interesting qualitative features of these terms are that $\left\langle T_{00}\right\rangle_{t}$ vanishes for minimal coupling, whereas the spatial components $\left\langle T_{j k}\right\rangle_{t}$ vanish for $\xi=\frac{1}{4}$.

For example, in the two-dimensional case we have

$$
\left\langle T_{00}\right\rangle_{t}=\frac{\xi}{\pi}\left[t^{2}+4(j a-x)^{2}+4(k b-y)^{2}\right]^{-5 / 2}\left[2 t^{2}-4(j a-x)^{2}-4(k b-y)^{2}\right] .(12
$$

Here $a$ and $b$ are the side lengths of the rectangle, and $j$ and $k$ are the indices characterizing the image point. Equation (12) applies if the sides of the box are all Dirichlet or all Neumann; in more general cases, the overall sign is determined by the parity of the number of Dirichlet sides struck by the path. 


\subsection{Side paths}

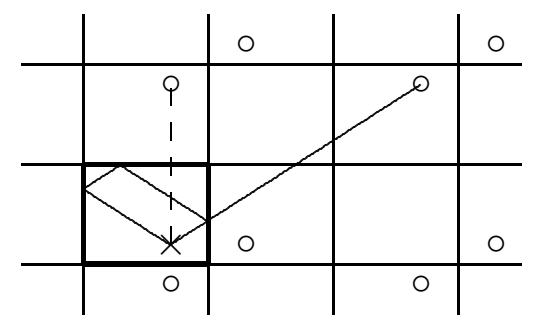

Regularized side terms will be our test case for the distributional renormalized Einstein equation. Only the shortest, perpendicular cases are divergent.

For $d=2$ we find

$$
\begin{aligned}
\pm\left\langle T_{00}\right\rangle_{t}= & -\frac{1}{4 \pi}\left[t^{2}+4(j a-x)^{2}+4(k b)^{2}\right]^{-5 / 2}\left[2 t^{2}-4(j a-x)^{2}-4(k b)^{2}\right] \\
& -\frac{\xi-\frac{1}{4}}{\pi}\left[t^{2}+4(j a-x)^{2}+4(k b)^{2}\right]^{-5 / 2}\left[t^{2}-4(j a-x)^{2}+4(k b)^{2}\right],
\end{aligned}
$$

where \pm applies for all Dirichlet sides and all Neumann sides, respectively. In three dimensions we present the case $\xi=\frac{1}{4}$ and $k, l, j=0$ (i.e., one of the short paths):

$$
\left\langle T_{00}\right\rangle_{t}= \pm \frac{1}{2 \pi^{2}} \frac{4 x^{2}-3 t^{2}}{\left(t^{2}+4 x^{2}\right)^{3}} .
$$

In this case, unlike the corner case, the integral over $x$ at nonzero $t$ does not vanish, but it is still finite; it is proportional to $t^{-3}$, hence divergent if the cutoff is removed.

\section{The gravitational implications}

\subsection{The linearized Einstein equation}

In the notation of Schutz [7], the linearization of (2) is

$$
-16 \pi T_{\mu \nu}=\partial_{\alpha} \partial^{\alpha} \bar{h}_{\mu \nu}, \quad \bar{h}_{\mu \nu} \equiv h_{\mu \nu}-\frac{1}{2} h_{\alpha}^{\alpha} \eta_{\mu \nu}, \quad h_{\mu \nu} \equiv g_{\mu \nu}-\eta_{\mu \nu},
$$

with the gauge $\partial_{\alpha} \bar{h}_{\mu}^{\alpha}=0$. We assume a static situation and let $\rho \equiv T_{00}, h \equiv \bar{h}_{00}$. If the source (13) exists in the region of positive $x$, the main component of (14) is

$$
-\nabla^{2} h=16 \pi \rho= \pm \frac{8}{\pi} \frac{4 x^{2}-3 t^{2}}{\left(t^{2}+4 x^{2}\right)^{3}} \theta(x)
$$

$\theta$ being the unit step function. (We are agnostic for the moment about what exists on the negative side of the wall, except that its influence could be combined linearly with that of our source.)

Now assume that the wall is infinite in extent, so that the natural solution of (15) will be a function of $x$ only. Then $\nabla^{2} h=\frac{d^{2} h}{d x^{2}}$. The solution that vanishes for negative $x$ is

$$
h(x)= \pm \frac{\theta(x)}{\pi}\left[\frac{4 x}{t^{3}} \tan ^{-1}\left(\frac{2 x}{t}\right)-\frac{1}{t^{2}+4 x^{2}}+\frac{1}{t^{2}}\right] .
$$

If we take the limit $t \downarrow 0$ in the equation (15), we get a differential equation with a distribution as source. If we take the limit $t \downarrow 0$ in the solution (16), we get a singular 
distribution. Both limits involve somewhat arbitrary regularizations (Hadamard finite parts). We expect, first of all, that the limit solution solves the limit equation; this should be an automatic consequence of the continuity of differential operators acting in distribution spaces. We also hope that the result is a plausible renormalized model for a system with a physically realistic cutoff. We shall next describe preliminary results that seem to verify these expectations. (The one-dimensional case was briefly reported in $[16]$.)

\subsection{Distributional asymptotics}

The necessary distributional tools are found in the book of Estrada and Kanwal [17]:

Moment Expansion Theorem: Let $f \in \mathcal{S}^{\prime}(\mathbf{R})$ with support bounded on the left. Suppose

$$
f(x)=b_{1} x^{\beta_{1}}+\cdots+b_{n} x^{\beta_{n}}+\mathcal{O}\left(x^{\beta}\right), \quad \text { as } x \rightarrow \infty,
$$

where $\beta_{1}>\beta_{2}>\cdots>\beta_{n}>\beta$, and $-(k+1)>\beta>-(k+2)$. Then as $\lambda \rightarrow \infty$,

$$
f(\lambda x)=\sum_{j=1}^{n} b_{j} g_{j}(\lambda x)+\sum_{j=0}^{k}(-1)^{j} \mu_{j} \delta^{(j)}(\lambda x) / j !+\mathcal{O}\left(\lambda^{\beta}\right)
$$

in the space $\mathcal{S}^{\prime}(\mathbf{R})$, where $g_{j}(x)=x^{\beta_{j}} \theta(x)$ if $\beta_{j} \neq-1,-2,-3, \ldots$ and $g_{j}(x)=$ $\mathcal{P f}\left(x^{\beta_{j}} \theta(x)\right)$ if $\beta_{j}=-1,-2,-3, \ldots$. Here the moments are

$$
\mu_{j}(f)=\text { F.P. } \int_{-\infty}^{\infty} f(x) x^{j} d x .
$$

("F.P." stands for "finite part" and refers to integrals. "Pf" stands for "pseudofunction" and refers to distributions defined by a finite-part prescription.)

An intuitive verbal summary of the theorem is this: When the distribution $f(\lambda x)$ is applied to a test function $\phi$, it is legitimate under certain technical conditions to expand $\phi(x)$ in a Taylor series around $x=0$ and then to take the limit $\lambda \rightarrow+\infty$ term by term. In our application, $\lambda$ is $t^{-1}$.

\section{Other formulas:}

$$
\begin{aligned}
& \frac{d}{d x}[\theta(x) \ln x]=\mathcal{P f}\left(\frac{\theta(x)}{x}\right) . \\
& \frac{d}{d x} \mathcal{P} f\left(\frac{\theta(x)}{x^{k}}\right)=-k \mathcal{P f}\left(\frac{\theta(x)}{x^{k+1}}\right)+\frac{(-1)^{k} \delta^{(k)}(x)}{k !} . \\
& \mathcal{P f}\left(\frac{\theta(\lambda x)}{(\lambda x)^{d+1}}\right)=\frac{1}{\lambda^{d+1}} \mathcal{P f}\left(\frac{\theta(x)}{x^{d+1}}\right)+\frac{(-1)^{d} \ln \lambda \delta^{(d)}(x)}{\lambda^{d+1} d !} . \\
& \delta^{(j)}(\lambda x)=\lambda^{-(j+1)} \delta^{(j)}(x) .
\end{aligned}
$$

It is not immediately obvious how a function $f$ defines a distribution (also called $f$ ) if the resulting integral $\int f \phi$ is divergent. As recently reviewed in [18], there is a consistent way to define such a distribution (called $\mathcal{P} f f$ ) by discarding the leading 
divergent power terms in the asymptotics of $f$ at the origin; generality is recovered by replacing these terms with derivatives of the Dirac delta distribution with arbitrary finite coefficients. (Compare the treatment of renormalization in [19].) Formulas (17) and (18) show that the definition of the pseudofunctions is, for the most part, consistent with differentiation; however, (19) reflects the fact that the definition cannot be made scale-invariant, and the resulting $\ln \lambda$ term corresponds to the $\ln t$ ambiguity often encountered in renormalization in physics.

In order to evaluate the $t \rightarrow 0\left(\lambda=\frac{1}{t} \rightarrow \infty\right)$ behavior, we rewrite (15) and (16) as

$$
\begin{aligned}
& -\frac{d^{2} h}{d x^{2}}= \pm \frac{8}{\pi} \lambda^{4} \frac{4 \frac{x^{2}}{t^{2}}-3}{\left(1+4 \frac{x^{2}}{t^{2}}\right)^{3}} \theta(x), \\
& h(x)= \pm \frac{\theta(x)}{\pi} \lambda^{2}\left[4 \frac{x}{t} \tan ^{-1}\left(2 \frac{x}{t}\right)-\frac{1}{1+4 \frac{x^{2}}{t^{2}}}+1\right] .
\end{aligned}
$$

For the evaluation of the asymptotic behavior of (21), the relevant distribution for the moment expansion theorem is

$$
f_{1}(x)=\frac{4 x^{2}-3}{\left(1+4 x^{2}\right)^{3}} \theta(x)=\frac{1}{16} \frac{1}{x^{4}}+\mathcal{O}\left(\frac{1}{x^{6}}\right) \quad \text { as } x \rightarrow \infty .
$$

The moment expansion theorem therefore states, up to the relevant order, that

$$
\begin{aligned}
f_{1}(\lambda x) \sim & \frac{1}{16} \mathcal{P f}\left(\frac{\theta(\lambda x)}{(\lambda x)^{4}}\right)+\sum_{j=0}^{3}(-1)^{j} \mu_{j}\left(f_{1}\right) \frac{\delta^{(j)}(\lambda x)}{j !}+\mathcal{O}\left(\frac{1}{\lambda^{5}}\right) \\
= & \frac{1}{16}\left\{\frac{1}{\lambda^{4}} \mathcal{P f}\left(\frac{\theta(x)}{x^{4}}\right)-\frac{\ln \lambda \delta^{\prime \prime \prime}(x)}{3 ! \lambda^{4}}\right\} \\
& +\sum_{j=0}^{3}(-1)^{j} \mu_{j}\left(f_{1}\right) \frac{\delta^{(j)}(x)}{j ! \lambda^{j+1}}+\mathcal{O}\left(\frac{1}{\lambda^{5}}\right),
\end{aligned}
$$

the moments $\mu_{j}\left(f_{1}\right)$ of the function $f_{1}(x)$ being

$$
\begin{aligned}
& \mu_{0}\left(f_{1}\right)=\int_{0}^{\infty} \frac{4 x^{2}-3}{\left(1+4 x^{2}\right)^{3}} d x=-\frac{\pi}{4}, \\
& \mu_{1}\left(f_{1}\right)=\int_{0}^{\infty} \frac{4 x^{2}-3}{\left(1+4 x^{2}\right)^{3}} \cdot x d x=-\frac{1}{8}, \\
& \mu_{2}\left(f_{1}\right)=\int_{0}^{\infty} \frac{4 x^{2}-3}{\left(1+4 x^{2}\right)^{3}} \cdot x^{2} d x=0, \\
& \mu_{3}\left(f_{1}\right)=\text { F.P. } \int_{0}^{\infty} \frac{4 x^{2}-3}{\left(1+4 x^{2}\right)^{3}} \cdot x^{3} d x=-\frac{3}{32}+\frac{1}{16} \ln 2 .
\end{aligned}
$$

Therefore, the distributional limit of the differential equation (15) is

$$
\begin{aligned}
-\frac{d^{2} h(x)}{d x^{2}}= & \pm \frac{1}{2 \pi} \mathcal{P f}\left(\frac{\theta(x)}{x^{4}}\right) \mp 2 \lambda^{3} \delta(x) \pm \frac{1}{\pi} \lambda^{2} \delta^{\prime}(x) \pm \frac{1}{8 \pi} \delta^{\prime \prime \prime}(x) \\
& \mp \frac{1}{12 \pi} \ln (2 \lambda) \delta^{\prime \prime \prime}(x)+\mathcal{O}\left(\frac{1}{\lambda}\right) .
\end{aligned}
$$


Vacuum Energy Density and Gravity

We continue with the analysis of $h(x)$ as given in (22). The relevant functions this time are

$$
\begin{aligned}
& f_{2}(x)=\frac{1}{1+4 x^{2}} \theta(x)=\frac{1}{4 x^{2}}+\mathcal{O}\left(\frac{1}{x^{4}}\right) \quad \text { as } x \rightarrow \infty \\
& f_{3}(x)=x \tan ^{-1}(2 x) \theta(x)=\frac{\pi}{2} x-\frac{1}{2}+\frac{1}{24} \frac{1}{x^{2}}+\mathcal{O}\left(\frac{1}{x^{4}}\right) \quad \text { as } x \rightarrow \infty .
\end{aligned}
$$

The moment expansion theorem says that

$$
\begin{aligned}
f_{2}(\lambda x) \sim & \frac{1}{4} \mathcal{P f}\left(\frac{\theta(\lambda x)}{(\lambda x)^{2}}\right)+\sum_{j=0}^{1}(-1)^{j} \mu_{j}\left(f_{2}\right) \frac{\delta^{(j)}(\lambda x)}{j !}+\mathcal{O}\left(\frac{1}{\lambda^{3}}\right) \\
= & \frac{1}{4 \lambda^{2}} \mathcal{P f}\left(\frac{\theta(x)}{x^{2}}\right)-\frac{1}{4 \lambda^{2}} \ln \lambda \delta^{\prime}(x) \\
& +\sum_{j=0}^{1}(-1)^{j} \mu_{j}\left(f_{2}\right) \frac{\delta^{(j)}(x)}{j ! \lambda^{j+1}}+\mathcal{O}\left(\frac{1}{\lambda^{3}}\right),
\end{aligned}
$$

and

$$
\begin{aligned}
f_{3}(\lambda x) \sim & \frac{\pi}{2} \theta(\lambda x)(\lambda x)-\frac{1}{2} \theta(\lambda x)+\frac{1}{24} \mathcal{P f}\left(\frac{\theta(\lambda x)}{(\lambda x)^{2}}\right) \\
& +\sum_{j=0}^{1}(-1)^{j} \mu_{j}\left(f_{3}\right) \frac{\delta^{(j)}(\lambda x)}{j !}+\mathcal{O}\left(\frac{1}{\lambda}\right) \\
= & \frac{\pi}{2} \lambda \theta(x) x-\frac{1}{2} \theta(x)+\frac{1}{24 \lambda^{2}} \mathcal{P} f\left(\frac{\theta(x)}{x^{2}}\right)-\frac{1}{24} \frac{1}{\lambda^{2}} \ln \lambda \delta^{\prime}(x) \\
& +\sum_{j=0}^{1}(-1)^{j} \mu_{j}\left(f_{3}\right) \frac{\delta^{(j)}(x)}{j ! \lambda^{j+1}}+\mathcal{O}\left(\frac{1}{\lambda}\right) .
\end{aligned}
$$

The relevant moments this time are

$$
\begin{aligned}
& \mu_{0}\left(f_{2}\right)=\int_{0}^{\infty} \frac{1}{1+4 x^{2}} d x=\frac{\pi}{4}, \\
& \mu_{1}\left(f_{2}\right)=\text { F.P. } \int_{0}^{\infty} \frac{1}{1+4 x^{2}} x d x=\frac{1}{4} \ln 2, \\
& \mu_{0}\left(f_{3}\right)=\text { F.P. } \int_{0}^{\infty} x \tan ^{-1}(2 x) d x=\frac{\pi}{16}, \\
& \mu_{1}\left(f_{3}\right)=\text { F.P. } \int_{0}^{\infty} x^{2} \tan ^{-1}(2 x) d x=\frac{1}{72}+\frac{1}{24} \ln 2 .
\end{aligned}
$$

Forming the appropriate linear combination of these contributions, one obtains

$$
\begin{aligned}
h(x)= & \pm 2 \lambda^{3} \theta(x) x \mp \frac{1}{\pi} \lambda^{2} \theta(x) \mp \frac{1}{12 \pi} \mathcal{P} f\left(\frac{\theta(x)}{x^{2}}\right) \\
& \mp \frac{1}{18 \pi} \delta^{\prime}(x) \pm \frac{1}{12 \pi} \ln (2 \lambda) \delta^{\prime}(x) .
\end{aligned}
$$

Taking the second derivative of (27) according to the rules (17)-(19), we find that the equation (24) is indeed satisfied. 


\subsection{Renormalization}

It is now possible to replace $\ln \lambda$ and positive powers of $\lambda$ by arbitrary constants in a consistent way throughout the formulas. Such constants should be lumped into properties of the boundary material. The result is a consistent renormalized Einstein equation for a scalar field with Dirichlet or Neumann condition on a plane. (A physically complementary treatment of mass renormalization is reported in the papers of Milton and Shajesh in this volume.)

To reiterate: In the absence of a detailed microscopic model of the boundary material, formally infinite terms should be replaced by finite terms with the same geometrical form and unknown coefficients that must be fixed experimentally. This is the traditional philosophy of renormalization in fundamental quantum field theory, where divergences are attributed to ignorance of ultrahigh-energy physics (e.g., [20]). There is a difference (largely psychological, in our opinion) between the two situations that makes some people hesitant to accept this analogy: In relativistic quantum field theories, cutoff theories are always physically unacceptable for some reason (lack of Lorentz invariance, nonlocality, indefinite metric in Hilbert space, ... ); so one believes that the renormalized ideal theory is closer to the truth. In Casimir theory, in contrast, one believes the idealized theory is a defective approximation to one that models the boundary materials (especially their interaction with high-frequency modes) realistically; so cutoff theories are believed to be closer to the truth. Certainly, an idealized theory that is more than a toy model must ultimately pass a test of experimental relevance.

\subsection{Outlook}

The next step is to establish (probably by soft general arguments rather than explicit calculation of moments) that the distributional theory of the Einstein equation extends to curved boundaries, and also to the edges and corners of the parallelepiped. One would also like to handle the more physical case of the electromagnetic field, and then extend the treatment to dielectric boundaries. More ambitiously, we would like to deal with a curved background space, and ultimately consider branes as boundaries.

\section{References}

[1] Milton K A, Kantowski R, Kao C and Wang Y 2001 Mod. Phys. Lett. A 16 2281-2289

[2] Elizalde E 2007 J. Phys. A: Math. Gen. 40 6647-6655

[3] Saharian A A 2006 Phys. Rev. D 73064019

[4] Deutsch D and Candelas P 1979 Phys. Rev. D 20 3063-3080

[5] Schaden M 2006 Phys. Rev. A 73042102

[6] Barton G 2001 J. Phys. A: Math. Gen. 34 4083-4114

[7] Schutz B F 1985 A First Course in General Relativity (Cambridge: Cambridge)

[8] Lebedev S L 1996 Zh. Eksp. Teor. Fiz. 110 769-792 [JETP 83 423-434]

[9] Fulling S A 2003 J. Phys. A: Math. Gen. 36 6857-6873

[10] Saharian A A 2004 Phys. Rev. D 69085005

[11] Cavero-Peláez I, Milton K A and Wagner J 2006 Phys. Rev. D 73085004 
[12] Cavero-Peláez I, Milton K A and Kirsten K 2007 J. Phys. A: Math. Gen. 40 3607-3631

[13] Hertzberg M P, Jaffe R L, Kardar M and Scardicchio A 2007 Phys. Rev. D 76045016

[14] Balian R and Bloch C 1972 Ann. Phys., NY 69 76-160

[15] Ford L H and Svaiter N F 1998 Phys. Rev. D 58065007

[16] Fulling S A 2004 Quantum Field Theory Under the Influence of External Conditions ed K A Milton (Princeton, NJ: Rinton) pp 166-174

[17] Estrada R and Kanwal R P 2002 A Distributional Approach to Asymptotics: Theory and Applications (Boston: Birkhäuser) [2nd ed. of Asymptotic Analysis: A Distributional Approach (Boston: Birkhäuser) 1994]

[18] Estrada R and Fulling S A 2002 J. Phys. A: Math. Gen. 35 3079-3089

[19] Bogoliubov N N and Shirkov D V 1959 Introduction to the Theory of Quantized Fields (New York: Wiley)

[20] Zee A 2003 Quantum Field Theory in a Nutshell (Princeton: Princeton) 